\title{
Pituitary Abscess: A Rare Clinical Entity in the Presence of Acute Rhinosinusitis: A Case Report
}

\author{
Vincent John Anagnos ${ }^{1}$ Ricardo A. Hanel ${ }^{2}$ Iman Naseri ${ }^{3}$ \\ ${ }^{1}$ Lake Erie College of Osteopathic Medicine, Bradenton Campus, \\ Bradenton, Florida, United States \\ 2 Department of Neurosurgery, Baptist Medical Center Jacksonville, \\ Jacksonville, Florida, United States \\ ${ }^{3}$ Department of Surgery - Otolaryngology Head \& Neck Surgery,

\begin{abstract}
Address for correspondence Iman Naseri, MD, FACS, Department of Surgery - Otolaryngology Head \& Neck Surgery, University of Florida College of Medicine - Jacksonville, 6817 Southpoint Parkway, Suite 502, Jacksonville, FL 32216, United States

(e-mail: naseri@nfsinus.com).
\end{abstract} University of Florida College of Medicine - Jacksonville, Jacksonville, Florida, United States

J Neurol Surg Rep 2018;79:e36-e40.

\begin{abstract}
A pituitary abscess is an often-overlooked diagnosis in the clinical presentation of a sellar mass. Due to its rare incidence and nonspecific presentation, diagnosis and treatment is often delayed. The authors describe a 56-year-old male patient presenting with acute onset of severe headache, visual field deficit, and radiologic findings of an expansile sellar lesion. The presenting symptoms were unremarkable for the diagnosis of meningitis, cavernous sinus thrombosis, and septicemia. Recent medical history included symptoms of rhinosinusitis on the days preceding his acute presentation. The

\section{Keywords}

- pituitary abscess

- rhinosinusitis

- acute sinusitis

- hypopituitarism

- staphylococcal epidermidis

- pituitary apoplexy

- brain abscess initial clinical presentation was suggestive of a possible pituitary apoplexy. Intraoperative findings revealed purulent output upon surgical entry of the sella. Histopathology confirmed the diagnosis of a pituitary abscess. Review of the clinical and radiologic data revealed evidence of multiple opacifications within the paranasal sinuses, along with dehiscence overlying the sellar bone, supporting a diagnosis of secondary pituitary abscess. This case, along with a review of the available literature, will serve to expand our knowledge of this rare disease process that is often overlooked. Clinicians should be mindful of this condition, and include a primary versus secondary pituitary abscess in the differential workup on such cases.
\end{abstract}

\section{Introduction}

Pituitary abscess is a rare yet life-threatening cause of a sellar mass. The diagnosis makes up less than $1 \%$ of all pituitary lesions. The clinical symptoms and radiographic findings of a pituitary abscess are similar to multiple other conditions such as pituitary adenoma, pituitary apoplexy, Rathke's cleft cyst, and craniopharyngioma. For this reason, it is rarely included in the initial differential diagnosis upon its presentation as a mass within the sellar region. ${ }^{1}$

The major predisposing risk factor for the development of a pituitary abscess is history of prior trans-sphenoidal sur-

received

September 21, 2017

accepted after revision

February 27, 2018
DOI https://doi.org/

10.1055/s-0038-1656531. ISSN 2193-6358. gery. ${ }^{2,3}$ Other commonly implicated risk factors include primary pituitary disease, previous irradiation to the sellar region, and concurrent presence of a systemic infection. An acute sinus infection spreading to the pituitary region has been documented as a cause for pituitary abscess formation, and it is often a result of direct extension from the sphenoid sinus. ${ }^{4-6}$

We present the case of a 56-year-old male with a pituitary abscess in concomitant presence of acute sinusitis. Intraoperatively, a provisional diagnosis of primary versus secondary pituitary abscess was made as purulent material was encountered upon opening of the dura.
License terms Stuttgart · New York

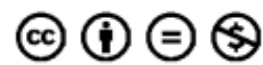




\section{Case Report}

A 56-year-old Caucasian male presented to an outside emergency room (ER) with acute onset of severe headache and intermittent visual field deficit. He was initially treated for migraines, which failed to improve his symptoms. A noncontrasted computed tomography (CT) scan of the head performed in an outside emergency department revealed a soft tissue, $18 \times 22 \mathrm{~mm}$ mass occupying the sellar region with superior extension and mass-effect upon the optic chiasm. The patient was then transferred to a tertiary hospital with neurovascular specialty care. The patient presented to the hospital with stable vital signs, and without a history of fevers, nausea, vomiting, or any other cranial nerve deficits in addition to his blurred vision. His initial laboratory workup was overall unremarkable, but did show signs of anterior pituitary dysfunction (-Table $\mathbf{1}$ ).

Nine days prior to his presentation, he was seen by his primary care physician for complaints related to a mild headache and nasal congestion with sinus pressure. He had no fever and denied visual changes at that time. He was given a course of doxycycline and a topical nasal steroid spray to use as empiric therapy for a suspected sinus infection. His past medical history includes obesity, hypertension, hyperlipidemia, and rheumatoid arthritis, requiring weekly etanercept injections. He had no prior surgical history and denied any prior history of neurological, sinonasal, or endocrine conditions. He denied any past history of head trauma, intravenous drug use, and any recent fevers, chills, or signs of a systemic infection. Upon his intensive care unit (ICU) admission, magnetic resonance imaging (MRI) of the brain revealed a $2.0 \times 2.1 \times 2.2 \mathrm{~cm}$ sellar and suprasellar ringenhancing lesion displaying mass effect on the optic chiasm (-Fig. 1). These findings were consistent with a pituitary macroadenoma, and the differential diagnosis included pituitary apoplexy versus a Rathke's cleft cyst. The patient was referred to neurosurgery and otolaryngology for surgical management.

Table 1 Preoperative biochemistry laboratory values

\begin{tabular}{|l|l|l|}
\hline Laboratory test & Laboratory value & Normal range \\
\hline White blood cell & $7.69 \mathrm{~K} / \mathrm{mcL}$ & $4.5-11.0 \mathrm{~K} / \mathrm{mcL}$ \\
\hline Serum sodium & $135 \mathrm{mEq} / \mathrm{L}$ & $135-145 \mathrm{mEq} / \mathrm{L}$ \\
\hline Prolactin & $18.7 \mathrm{ng} / \mathrm{mL}$ & $3.0-13.0 \mathrm{ng} / \mathrm{mL}$ \\
\hline IGF-1 & $223 \mathrm{ng} / \mathrm{mL}$ & $50-317 \mathrm{ng} / \mathrm{mL}$ \\
\hline Growth hormone & $0.19 \mathrm{ng} / \mathrm{mL}$ & $0.01-1.0 \mathrm{ng} / \mathrm{mL}$ \\
\hline ACTH & $<5 \mathrm{pg} / \mathrm{mL}$ & $6.0-50.0 \mathrm{pg} / \mathrm{mL}$ \\
\hline Cortisol & $<0.4 \mathrm{mcg} / \mathrm{dL}$ & $8.7-22.4 \mathrm{mcg} / \mathrm{dL}$ \\
\hline Free T4 & $0.6 \mathrm{ng} / \mathrm{dL}$ & $0.5-1.4 \mathrm{ng} / \mathrm{dL}$ \\
\hline FSH & $4.4 \mathrm{mIU} / \mathrm{mL}$ & $1.0-8.0 \mathrm{mIU} / \mathrm{mL}$ \\
\hline LH & $\mathbf{0 . 5} \mathrm{mIU} / \mathrm{mL}$ & $1.2-8.0 \mathrm{mIU} / \mathrm{mL}$ \\
\hline
\end{tabular}

Abbreviations: ACTH, adrenocorticotropic hormone; $\mathrm{FSH}$, follicle-stimulating hormone; IGF, insulin-like growth factor; LH, luteinizing hormone.

Note: Abnormal values are in bold.
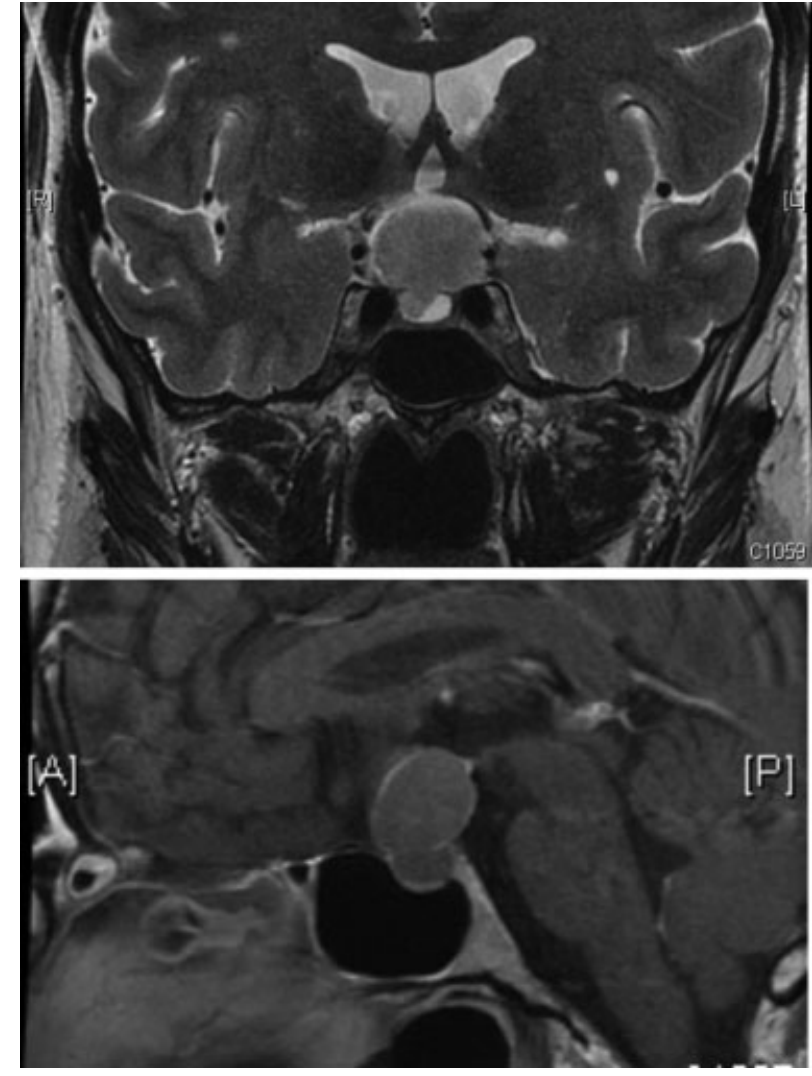

Fig. 1 A preoperative coronal T2-weighted magnetic resonance imaging (MRI) along with a preoperative midline sagittal T1-weighted MRI.

Intraoperatively, frank purulence was encountered immediately after the dural incision, leading to a diagnosis of primary versus secondary pituitary abscess. This would be further delineated by the results of the histopathology. The specimen was also sent for culture analysis. The surgical bed was irrigated and all of the purulence was evacuated meticulously. A low-flow cerebrospinal fluid (CSF) leak was encountered from the arachnoid region of the intrasellar space. The defect was repaired using a pedicled septonasal flap based on vascular supply of posterior septonasal artery. ${ }^{7}$

A postoperative MRI confirmed complete evacuation of the sellar contents ( - Fig. 2). The content collected from the abscess within the sella was cultured, revealing oxacillin-resistant Staphylococcal epidermidis. S. epidermidis is among the normal sinonasal flora, and it is commonly isolated from intracranial infections. $^{8-10}$ Blood and CSF cultures were negative. The patient was started on intravenous vancomycin and cefepime postoperatively, based on recommendations derived from consultation with infectious disease specialty. Histopathologic evaluation of the sellar contents contained normal pituitary tissue with fibrosis and areas of mild acute and chronic inflammation. There was no evidence of a pituitary adenoma, lymphocytic hypophysitis, granulomatous inflammation, or malignancy. The patient developed transient postoperative diabetes insipidus, treated with desmopressin replacement therapy until its resolution a few weeks later. He was discharged home with a peripherally inserted central catheter (PICC) line 


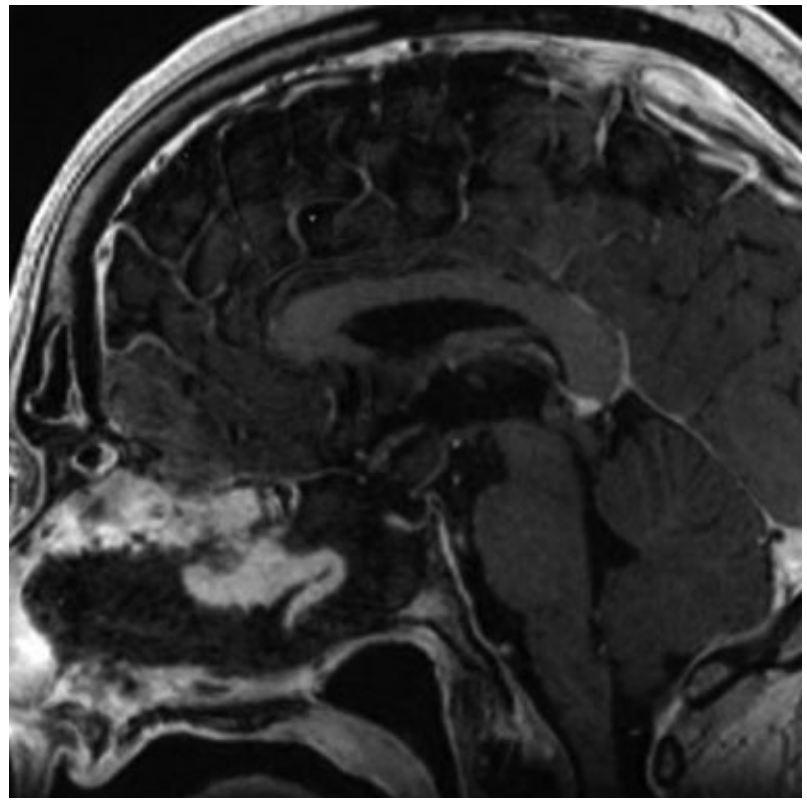

Fig. 2 Postoperative midline sagittal T1-weighted magnetic resonance imaging (MRI). The sellar mass has been completely evacuated.

and a 3-week course of intravenous vancomycin and cefepime on the fifth postoperative day.

Two days after discharge, the patient developed symptoms of clear rhinorrhea, and was readmitted for the management of CSF leak. He underwent surgical exploration, and revision repair of the surgical bed, along with lumbar drain placement. Newly obtained intraoperative CSF and sinonasal cultures were negative, and the patient denied any meningeal symptoms. After normal postoperative convalescence, the patient was discharged home to complete his antibiotic therapy. The patient has tolerated the treatment well, and has shown no sign of disease recurrence after 7 months of follow-up.

\section{Discussion}

A pituitary abscess is a rare and potentially life-threatening condition that is difficult to confirm solely on clinical presentation. It exists as a primary versus a secondary phenom- enon. A primary pituitary abscess is diagnosed after all possible sources of secondary infection have been ruled out. ${ }^{11}$ The challenge in diagnosing a pituitary abscess is often due to a clinical presentation that is similar to commonly found expansile processes involving the sella, such as a pituitary adenoma, pituitary apoplexy, Rathke's cleft cyst, and craniopharyngioma. ${ }^{12}$

In a disease-free state, the pituitary gland exists within the sella turcica in a sterile compartment, contiguous with the remainder of the intracranial contents. The most common reported risk factor for the development of a secondary pituitary abscess is prior history of trans-sphenoidal surgery. Moreover, any infectious or iatrogenic insult altering the sphenoid sinus or sellar region is considered a risk factor for the development of a secondary pituitary abscess. ${ }^{2,3,13}$

Differentiating among primary versus secondary in the presentation of a pituitary abscess can be challenging. Whether there is an underlying infection that contributes to the formation of a pituitary abscess remains unclear in many such cases. In this case, the patient did not have any symptoms that suggested a severe infection, such as meningitis, sepsis, or cavernous sinus thrombosis. However, his clinical and radiologic findings did support a concomitant diagnosis of acute rhinosinusitis ( $\mathbf{F i g . ~ 3}$ ). Interestingly, the sinus CT inflammatory changes did not extend within the sphenoid sinus, but bony dehiscence along the sella was seen, which was also confirmed intraoperatively ( - Fig. 4).

In an effort to rule out all possible secondary causes of this disease process, the authors further studied the patient's past medical history. The patient had no prior surgical history, but he did have a diagnosis of rheumatoid arthritis, which required weekly etanercept injections. A case of etanercept-induced immunosuppression leading to meningitis further complicated by brain abscess formation has been reported in the literature. Although possible, in this case it was deemed unlikely given the absence of signs and symptoms of meningitis. ${ }^{14}$ Other known contributors of pituitary abscess were ruled out by lack of findings in the presenting history, physical exam, imaging, microbiology, and pathology results. Thus, it may be assumed that the coexistence of acute rhinosinusitis along with the presence of a bony dehiscence along the sella may

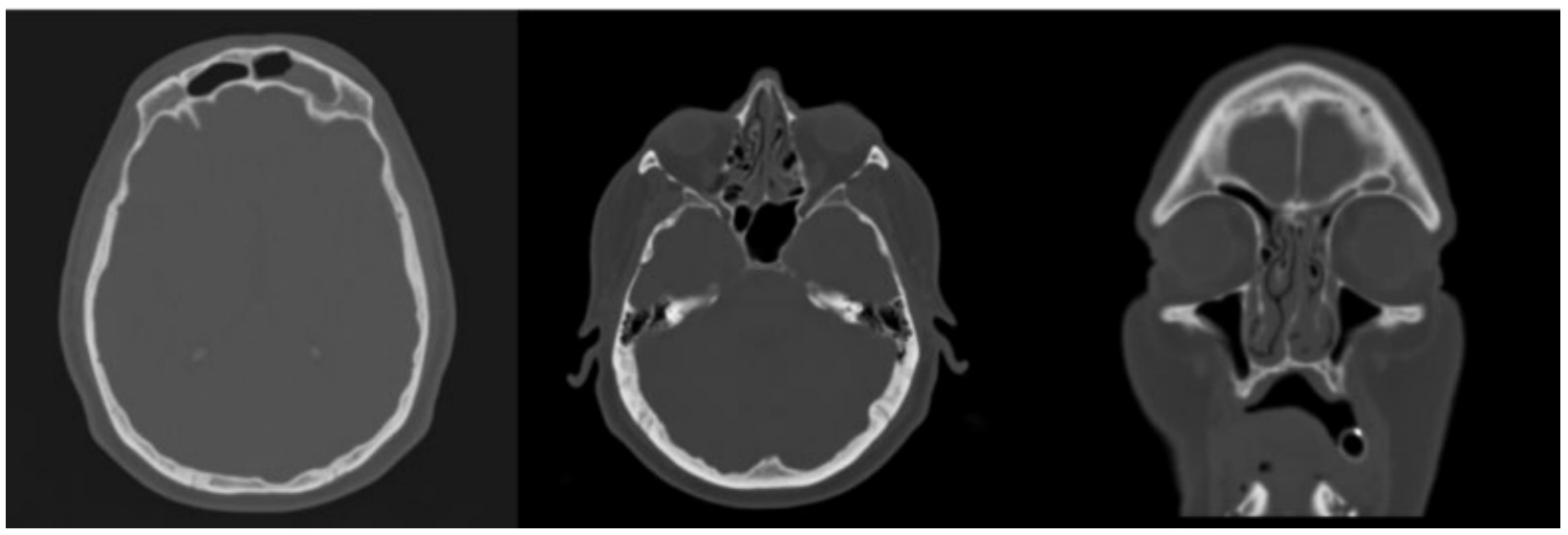

Fig. 3 A preoperative sinus computed tomography (CT) scan with two axial and one coronal view displaying bilateral frontal sinus opacification and patchy opacification of bilateral ethmoid sinuses. 


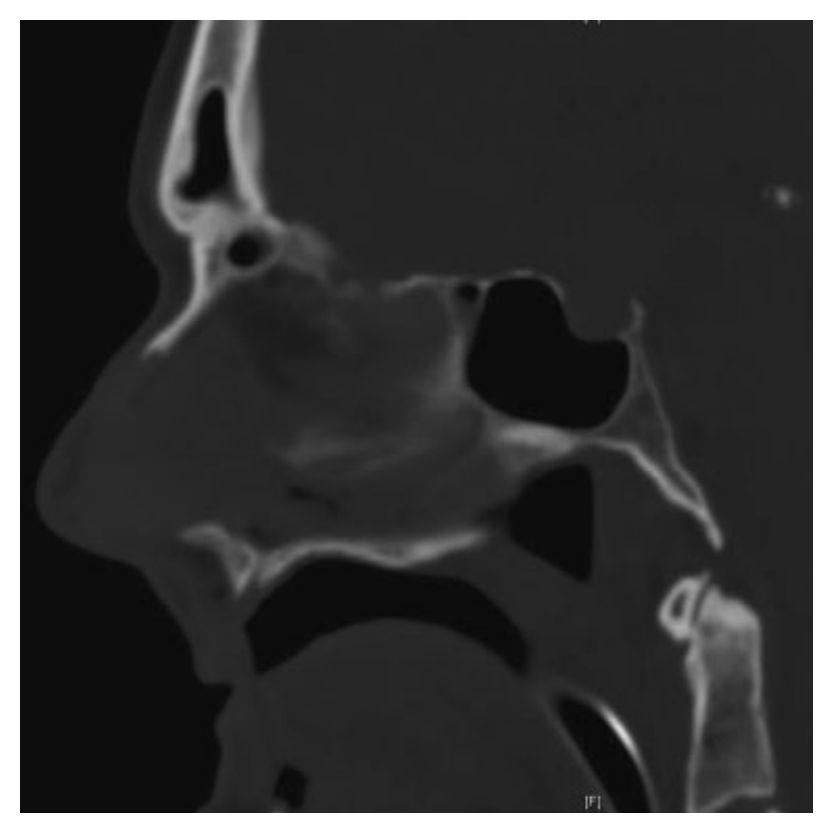

Fig. 4 A preoperative midline sagittal computed tomography (CT) scan concentrating on the sphenoid sinus displaying an absence of infiltration and apparent bony dehiscence of the bone overlying the anterior sella.

have predisposed this patient's disease formation, leading to a secondary pituitary abscess.

Our patient presented with severe headache and vision changes along with signs and symptoms of acute sinusitis and subclinical anterior pituitary dysfunction. According to the largest cohort study of pituitary abscesses reported to date (total of 66 patients), the five most common clinical presentations of a pituitary abscess includes: anterior pituitary dysfunction, headache, diabetes insipidus, visual disturbances, and symptoms related to an infection. ${ }^{1}$ Interestingly, our patient presented with four of these five characteristic findings, lacking only preoperative diabetes insipidus. Nonetheless, such symptoms are often ubiquitous among other more commonly encountered diagnoses such as a pituitary apoplexy and macroadenoma.

A variety of treatment modalities have been used in the management of both primary and secondary pituitary abscess. Currently, there are no predefined guidelines in the treatment regimen of such a diagnosis. While some authors have documented conservative treatment with intravenous antibiotics alone as an initial empiric treatment, ${ }^{15-17}$ the majority of cases are diagnosed and treated with surgical exploration. ${ }^{17-19}$ The rate of disease recurrence after surgery has shown to be less than $10 \%$ in several larger studies. ${ }^{1,2,17,19}$ However, larger abscesses may require a craniotomy approach for complete evacuation, and these cases have been associated with a higher recurrence rate, estimated at $17.2 \%{ }^{20}$ Overall, it is estimated that $66.1 \%$ of patients achieve at least partial recovery of pituitary function and $75.7 \%$ recover from associated visual deficits after treatment. ${ }^{20}$ Reported mortality rates have proven to be variable, but mortality was as high as $8.3 \%$ in one of the larger studies. ${ }^{2}$ The patient in this case had full resolution of his preoperative and postoperative pituitary dysfunction, along with resolution of his preoperative symptoms of headache and visual deficit.

This case uniquely illustrates the presentation and clinical management steps that led to the diagnosis of a secondary pituitary abscess. This report highlights the importance of the link between acute sinusitis in the presence of such a clinical presentation for a sellar lesion. Interestingly, the presence of a bony sellar dehiscence may have had a role in serving as a route for extension of the sinonasal infection to the intrasellar space. Therefore, the authors believe a high index of suspicion for a pituitary abscess is crucial upon the presentation of a sellar mass, especially in the setting of sinusitis or bony defects involving the sphenoid and sellar region. We recommend immediate surgical exploration in all such cases, and extended postoperative intravenous antibiotics if a pituitary abscess is found during exploration. Close outpatient follow-up is then required to ensure resolution of symptoms and any residual pituitary dysfunction. Additional reporting of such cases is encouraged to further elucidate the diagnosis and management of this rare clinical entity.

\section{Conflict of Interest \\ None.}

\section{References}

1 Gao L, Guo X, Tian R, et al. Pituitary abscess: clinical manifestations, diagnosis and treatment of 66 cases from a large pituitary center over 23 years. Pituitary 2017;20(02):189-194

2 Vates GE, Berger MS, Wilson CB. Diagnosis and management of pituitary abscess: a review of twenty-four cases. J Neurosurg 2001;95(02):233-241

3 Wang L, Yao Y, Feng F, et al. Pituitary abscess following transsphenoidal surgery: the experience of 12 cases from a single institution. Clin Neurol Neurosurg 2014;124:66-71

4 Dolan RW, Chowdhury K. Diagnosis and treatment of intracranial complications of paranasal sinus infections. J Oral Maxillofac Surg 1995;53(09):1080-1087

5 Shono T, Nishio S, Muratani H, Yasuda T, Fukui M, Moutai K. Pituitary abscess secondary to isolated sphenoid sinusitis. Minim Invasive Neurosurg 1999;42(04):204-206

6 Somali MH, Anastasiou AL, Goulis DG, Polyzoides C, Avramides A. Pituitary abscess presenting with cranial nerve paresis. Case report and review of literature. J Endocrinol Invest 2001;24(01): 45-50

7 Prevedello DM, Barges-Coll J, Fernandez-Miranda JC, et al. Middle turbinate flap for skull base reconstruction: cadaveric feasibility study. Laryngoscope 2009;119(11):2094-2098

8 Martín-Gandul C, Mayorga-Buiza MJ, Castillo-Ojeda E, et al. Sequential antimicrobial treatment with linezolid for neurosurgical infections: efficacy, safety and cost study. Acta Neurochir (Wien) 2016;158(10):1837-1843

9 Sharma N, Jaiswal AA, Banerjee PK, Garg AK. Complications of chronic suppurative otitis media and their management: a single institution 12 years experience. Indian J Otolaryngol Head Neck Surg 2015;67(04):353-360

10 Wu X, Liu Q Jiang X, Zhang T. Prevention options for ventriculoperitoneal shunt infections: a retrospective analysis during a fiveyear period. Int J Clin Exp Med 2015;8(10):19775-19780 
11 Hanel RA, Koerbel A, Prevedello DM, Moro MS, Araújo JC. Primary pituitary abscess: case report. Arq Neuropsiquiatr 2002;60 (3-B):861-865

12 Wolansky LJ, Gallagher JD, Heary RF, et al. MRI of pituitary abscess: two cases and review of the literature. Neuroradiology 1997;39(07):499-503

13 Lin BJ, Chen YH, Chung TT, Ma HI, Chen YH. Pituitary abscess following expanding sphenoid sinus pyocele: complication of endoscopic endonasal transsphenoidal surgery. Clin Neurol Neurosurg 2013;115(08):1502-1505

14 Kasirye Y, Epperla N, Manne JR, Bapani S, Garcia-Montilla RJ. Brain abscesses complicating acute pneumococcal meningitis during etanercept therapy. Clin Med Res 2012;10(04):230-235

15 Dechambenoit G, Datie A, Grunitzky EK, et al. Pituitary abscess, treated by medication [in French]. Rev Neurol (Paris) 1993;149 (10):567-571
16 Kaur A, Agrawal A, Mittal M. Presumed pituitary abscess without infectious source treated successfully with antibiotics alone. J Neuroophthalmol 2005;25(03):185-188

17 Zhu H, Gu XM, Hong J, Shen FX. Successful treatment of pituitary abscess with intravenous antibiotics: a case report and literature review. Genet Mol Res 2014;13(04):10523-10528

18 Liu F, Li G, Yao Y, et al. Diagnosis and management of pituitary abscess: experiences from 33 cases. Clin Endocrinol (Oxf) 2011; 74(01):79-88

19 Zhang X, Sun J, Shen M, et al. Diagnosis and minimally invasive surgery for the pituitary abscess: a review of twenty nine cases. Clin Neurol Neurosurg 2012;114(07):957-961

20 Agyei JO, Lipinski LJ, Leonardo J. Case report of a primary pituitary abscess and systematic literature review of pituitary abscess with a focus on patient outcomes. World Neurosurg 2017;101:76-92 